\title{
ADMINISTRACIÓN Y TERRITORIO EN ASTURIAS
}

\author{
Paz BENITO DEL POZO \\ Departamento de Geografia, Universidad de León
}

\section{INTRODUCCIÓN.}

En el contexto de una Geografía Política renovada en las últimas décadas, tras superarse el rechazo y las reticencias que mantuvieron a esta disciplina en un segundo plano como consecuencia del abuso ideológico que se hizo de sus contenidos en el período de entreguerras, y como parte de los temas que abarca en su formulación actual y más reciente (MÉNDEZ Y MOLINERO, 1984; SÁNCHEZ, 1992; TAYLOR, 1994; LÓPEZ TRIGAL Y BENITO DEL POZO, 1999), este artículo pretende ser una aportación a lo que podríamos llamar con toda propiedad una Geografía de la Administración, que tiene como objeto estudiar las relaciones entre el territorio y las distintas instancias de la Administración Pública, a diferentes escalas.

Según autores citados (LóPEZ TRIGAL y BeNITO DEL PozO, 1999:212), "los geógrafos tienen la misión de examinar los rasgos de la organización territorial y las características espaciales de cada sistema administrativo en sus diferentes niveles, comprender y analizar el fenómeno de los espacios administrativos, contando necesariamente con apoyo en otras disciplinas (...) Cabe entonces que los geógrafos estudien los rasgos de la Administración y sus repercusiones territoriales y sociopolíticas", tarea que viene a cubrir un importante hueco en la Geografía actual y a enriquecer la experiencia de los geógrafos en el análisis de las relaciones poder-espacio.

El enfoque que deberá prevalecer es el entendimiento de la política como gestión del territorio, asumiendo según la propuesta de J.-E. SÁNCHEZ (1992:71) el principio de la necesaria coherencia entre estructura y proceso social y estructura y proceso territorial pues "si no se dan unas formas adecuadas de articulación, se hará difícil alcanzar los objetivos políticos y sociales propuestos, dado que la articulación del territorio no permitirá su materialización".

Entre los temas que pueden ser objeto de esta Geografía de la Administración ocuparían un lugar destacado las bases de la organización administrativa del territorio, la relación entre los distintos niveles locales (municipal, comarcal), entre sí y con los niveles provincial, regional y nacional que pudieran darse, así como la intervención de los poderes públicos en el espacio a través de la planificación regional, el planeamiento urbanístico y la ordenación del territorio. No menos interés revisten el estudio de la distribución territorial de los presupuestos y su relación con la política de desarrollo regional asumida desde el poder, la provisión de servicios públicos, expresión en buena medida del compromiso de los respon-

Polígonos, nº 9, 1999, pp. 31-48. 
sables públicos con el desarrollo económico, social y territorial, o el análisis del empleo en el sector de la Administración.

Con respecto a la escala, si bien la estatal ha sido la preferida por numerosos geógrafos para analizar el alcance de las decisiones y actuaciones de los poderes públicos en materias como los transportes, la educación, la sanidad o la planificación del espacio, cada vez se impone con más fuerza el criterio de considerar las escalas regional y local como ámbitos privilegiados para la observación y análisis de fenómenos con una clara vinculación y dependencia del factor territorial (SÁNCHEZ, 1992; LÓPEZ TRIGAL Y BENITO DEL POZO, 1999).

\section{ORGANIZACIÓN ADMINISTRATIVO-TERRITORIAL DEL PRINCIPADO.}

El entramado institucional que caracteriza al Principado de Asturias, cuyo Estatuto de Autonomía fue aprobado por Ley Orgánica 7/1981 de 30 de diciembre y reformado por Ley Orgánica 1/1994 de 24 de marzo, manifiesta la complejidad propia de aquellas comunidades autónomas españolas que han accedido a la autonomía por la denominada "vía lenta" (Artículo 143 de la Constitución), lo que significa que tienen sin completar el proceso de trasferencia de competencias, a lo que debemos añadir su estructura uniprovincial. La consecuencia inmediata de esta situación es la confluencia sobre el territorio regional de tres niveles de gestión, si exceptuamos las instituciones europeas: Administración del Estado, Administración del Principado y Administraciones locales.

Las competencias del Principado, ampliadas tras la citada reforma del Estatuto de Autonomía en 1994, abarcan desde la organización de sus instituciones de autogobierno, pasando por la ordenación del territorio, urbanismo y vivienda, las obras públicas de interés regional, la pesca, la agricultura y ganadería, hasta el fomento del desarrollo económico, la investigación y la cultura o el apoyo y protección del bable. En suma, un abanico amplio pero incompleto de competencias que obliga a que el Principado y la Administración del Estado mantengan una estrecha colaboración que se concreta en numerosos convenios sobre materias tan diversas como infraestructuras, sanidad o promoción económica. Por su parte, la cooperación interregional es débil cuando no inexistente como lo demuestra el hecho de que no existen convenios firmados en ninguna materia con otras comunidades autónomas.

A escala local, las relaciones entre el Principado y los ayuntamientos se limitan, por parte del primero, a potenciar los municipios como entidad básica de la organización territorial, apoyando su labor a través de planes de cooperación para la recaudación de tributos, planes de cooperación en materia rural y asesoramiento jurídico, económico e informático. También se aprecia una actitud abierta del Principado hacia las mancomunidades, a las que apoya con subvenciones y cesión de maquinaria, como más adelante se verá.

Desde el punto de vista territorial, el Principado se organiza en municipios, que reciben el nombre de concejos. El número total de concejos asciende a 78. Si tenemos en cuenta el reducido tamaño de la región, en total $10.564 \mathrm{~km}^{2}$, es fácil deducir el alto grado de fragmentación en unidades concejiles, hecho $a$ 
priori poco favorable para tareas en las que el geógrafo puede estar en alguna medida involucrado como son la ordenación, la planificación y la gestión del territorio y que genera "disfuncionalidades serias, consumiendo unos presupuestos excesivos y muy mal aprovechados en medios humanos y materiales" (FERNÁNDEZ GARCÍA, 1989).

Por otra parte, aunque el Estatuto de Autonomía fija la necesidad de delimitar comarcas, Asturias, a diferencia de otras regiones españolas, carece de comarcas naturales, tradicionales o históricas reconocidas y hasta la fecha la comarca, como entidad local, no tiene rango jurídico. Ello no obsta para que desde principios de los años ochenta se hayan realizado diversas propuestas de comarcalización del territorio regional. En tal sentido, cabe destacar la realizada en 1985 por la Sociedad Asturiana de Estudios Económicos (SADEI), que delimitaba diez comarcas según el principio de similitud o analogía entre las unidades dadas, con el resultado que recoge la FIGURA 1.

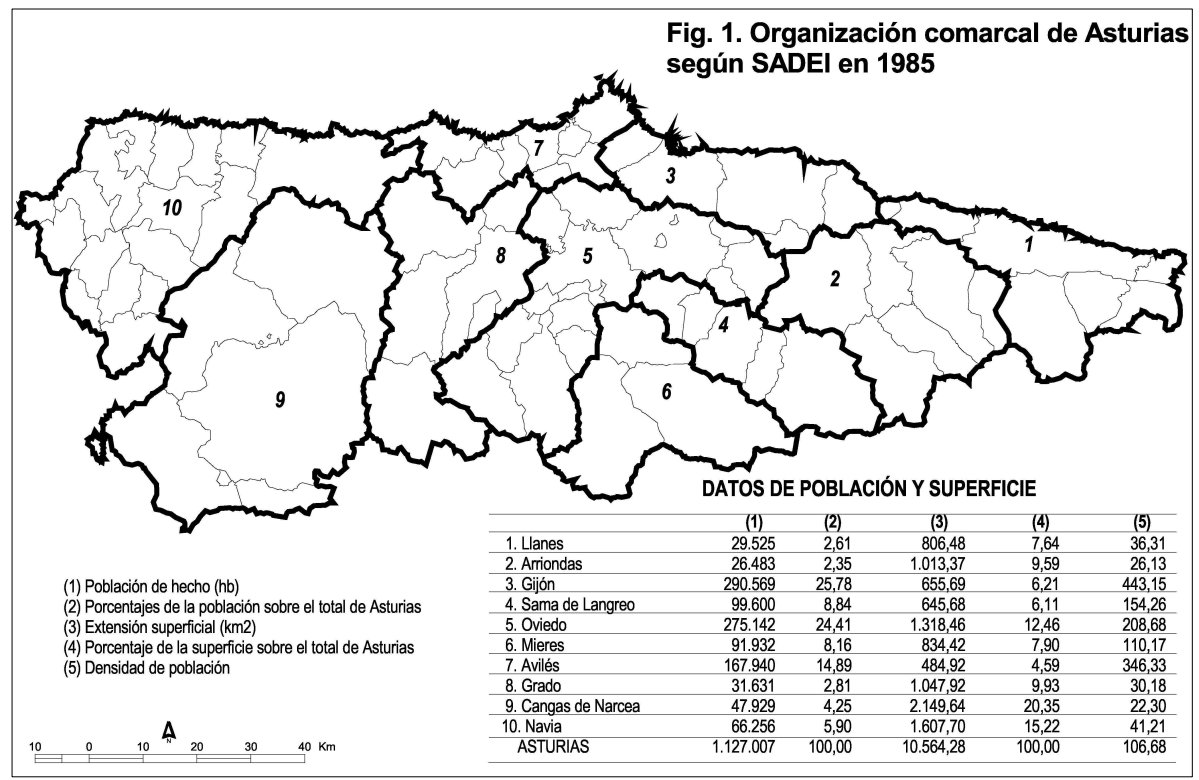

Tomando como punto de partida la comarcalización de Sadei, la Administración autonómica, a través de la Consejería de Ordenación del Territorio, Vivienda y Medio Ambiente, realizó en 1987 una propuesta concreta de demarcación territorial que se recoge en el documento Criterios para una demarcación territorial de Asturias, dirigido y coordinado por geógrafos de la Universidad de Oviedo. Según dicha propuesta, la región quedaría subdividida en tres grandes áreas subregionales designadas Occidente, Centro y Oriente y definidas como áreas de planificación; cada una de ellas se subdivide a su vez en comarcas, ocho en total frente a las diez que proponía Sadei, y éstas en territorios y áreas territoriales básicas (FIGURA 2). 


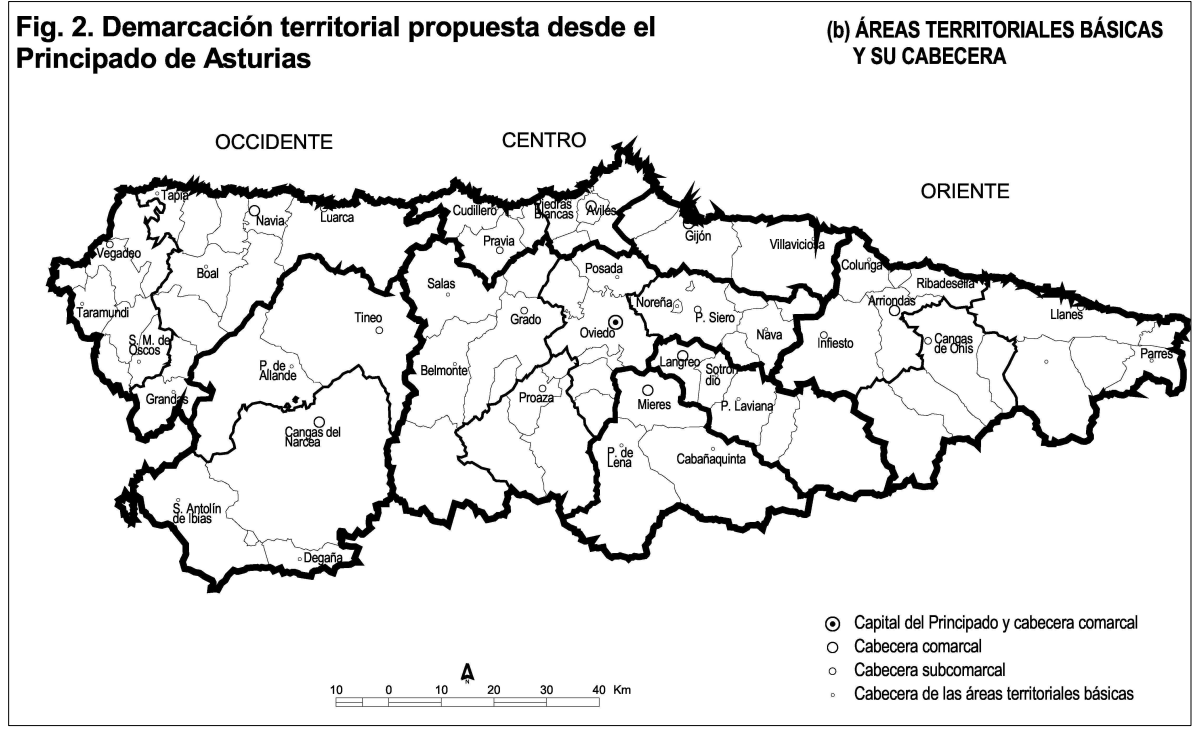

Asimismo, desde las figuras de planeamiento regional vigentes (Directrices Regionales de Ordenación del Territorio) se sigue fomentando la creación de comarcas (comarcas funcionales) con el objetivo último de definir ámbitos territoriales básicos para la ordenación de los diversos servicios y equipamientos públicos que permitan racionalizar el sistema de ciudades y villas, al tiempo que favorecen la planificación. En este sentido, las Directrices recogen la comarcalización que se presenta en la FIGURA 2 y a partir de este momento el Principado la asume a efectos prácticos, tal y como se refleja en los Presupuestos Generales $\mathrm{y}$ otros documentos de la Administración regional.

Sobre el acierto de esta comarcalización de facto se han apuntado algunas consideraciones (CASTELLS, 1994) tales como que el número de comarcas dentro de la Zona Central parece excesivo, siendo recomendable que las cuencas mineras del Caudal y Nalón quedasen englobadas en una sola comarca, al tiempo que se funden las de Oviedo, Gijón y Avilés, por razones de complementariedad funcional y rasgos de conjunto metropolitano; por el contrario, la comarcalización del oriente y el occidente aparece como eficaz.

La circunstancia arriba expuesta -falta de entidades locales de rango comarcal- explica, en buena medida, el nacimiento de asociaciones voluntarias de concejos en mancomunidades de servicios, fenómeno que ha dado origen a la formación desde 1985 de doce mancomunidades, con la particularidad de que esta forma de asociacionismo no implica pérdida de competencias para los ayuntamientos (FERNÁNDEZ GARCÍA, 1989). 


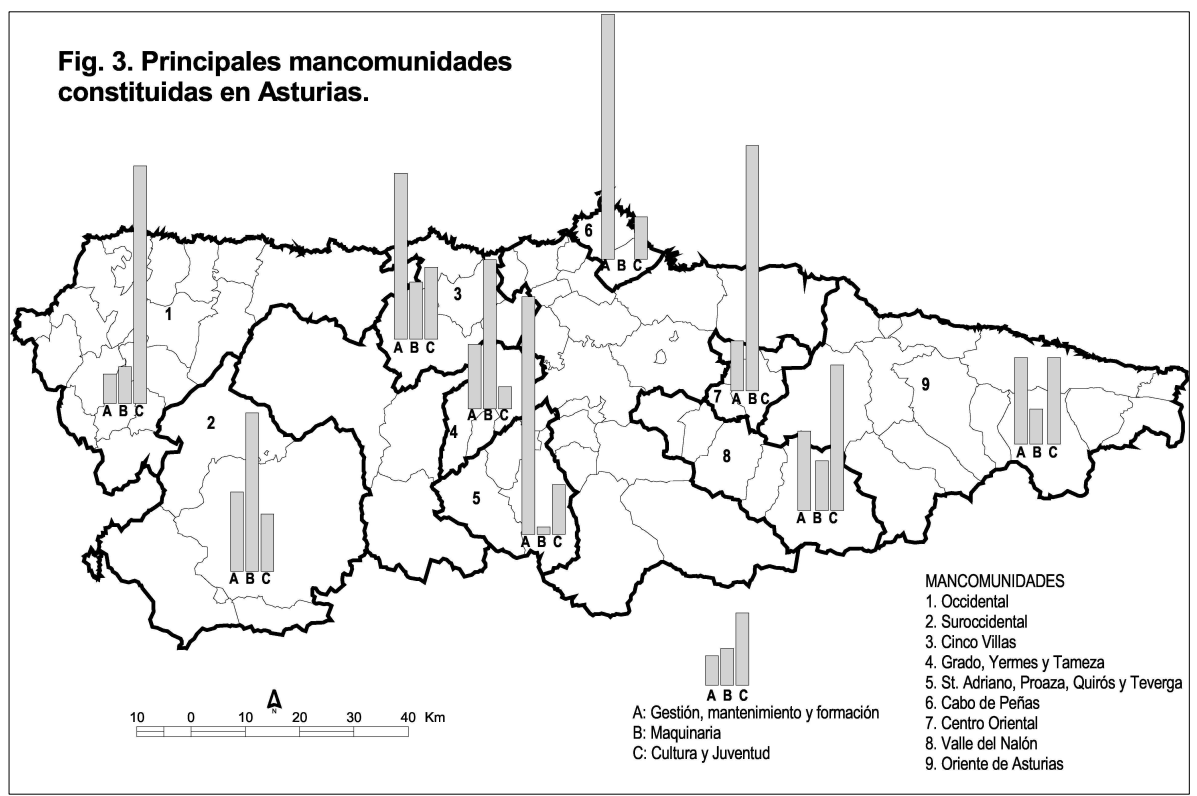

El débil funcionamiento, cuando no fracaso, de las mancomunidades actuales no resta eficacia a la figura en sí, que puede ser eficaz si se superan las reticencias localistas y la rivalidad entre las corporaciones implicadas por imponer sus particulares criterios e intereses.

Otra fórmula seguida en Asturias para resolver problemas de servicios que no pueden ser atendidos con eficacia desde la esfera local es la creación de consorcios. En efecto, allí donde los ayuntamientos y las mancomunidades no llegan, el Principado está propiciando la puesta en marcha de consorcios que atienden servicios a escala supramunicipal, e incluso regional, como son el abastecimiento de agua (CADASA), la recogida y tratamiento de los residuos sólidos urbanos (COGERSA) o la protección civil (CEISPA). Los consorcios son una figura a potenciar, no sólo por su probada eficacia, sino también porque actúan como factor de integración regional (BENITO DEL POZO, 1993; CASTELLS, 1994).

También el Estatuto de Autonomía contempla la posibilidad de crear Áreas Metropolitanas, aspecto de suma importancia habida cuenta de la existencia dentro del Área Central de Asturias de un sistema urbano de tipo metropolitano, articulado en torno a las ciudades de Oviedo, Gijón, Avilés, Mieres y Langreo, que en un futuro muy cercano puede requerir de una figura administrativa de carácter comarcal o supramunicipal desde la cual llevar a cabo la gestión y planificación de este espacio urbano que concentra a más del $80 \%$ de la población asturiana. El documento sobre Estrategias para la Reindustrialización de Asturias (Programa ERA), realizado a instancias de la Presidencia del Principado (CASTELLS, 1994) recoge el fenómeno metropolitano y propone su consolidación, incluyen- 
do los municipios que contienen las principales ciudades y sus adyacentes, dentro de los límites de lo que se conoce como Área Central.

Por último, en Asturias, como en Galicia, existe una entidad territorial de rango inferior al concejo, la parroquia rural, contemplada por el Estatuto de $\mathrm{Au}-$ tonomía en los términos siguientes: "se reconocerá personalidad jurídica a la parroquia rural como forma tradicional de convivencia y asentamiento de la población asturiana" (art. 6), aunque carece de una delimitación con validez legal. El sentido de la parroquia va más allá del mero carácter religioso. Como señala QUIRÓS LINARES (1993) "la parroquia tiene o ha tenido un fundamento económico en la existencia de montes comunales" y aunque se trata de una unidad territorial ínfima (por término medio cada concejo tiene unas once parroquias, dándose el caso de concejos con 54 parroquias) es operativa a efectos censales (Nomenclátor y Padrón Municipal de Habitantes) y catastrales, además de estar muy arraigada en la mentalidad colectiva y ser parte intrínseca de la identidad local. Estas razones justifican la tarea de delimitación llevada a cabo por el citado autor, lo que permite disponer de un mapa parroquial fiable.

\section{DISTRIBUCIÓN DE LOS PRESUPUESTOS GENERALES.}

Los Presupuestos Generales del Principado comprenden el presupuesto de la comunidad autónoma y los de sus organismos autonómicos. La evolución de su volumen acusa la crisis económica que sufre la región, que impone, como es obvio, restricciones al gasto público, a la vez que es reflejo del cambio político en el Gobierno del Principado (las elecciones de 1995 dieron la victoria al Partido Popular (PP) frente al Partido Socialista (PSOE), en el poder desde las primeras elecciones democráticas).

En efecto, en 1992 el valor total de los Presupuestos se elevó a 76.297,9 millones de pesetas, cifra que se fue incrementando en años sucesivos hasta llegar a 92.371,4 millones en 1995, año que marca el punto álgido del gasto en la década de los noventa pues a partir de dicho año se produce un recorte sustancial que sitúa los Presupuestos de 1996 en 70.377,6 millones de pesetas y los del año siguiente aún más abajo, 68.962,1 millones; el año 1998 representa un momento de recuperación y la cifra de los Presupuestos se alza hasta los 75.963,3 millones, sin que se logre el nivel alcanzado en el año 1992.

En el período expansivo 1992-1995 la Consejería más beneficiada fue la de Infraestructuras y Vivienda, en consonancia con los objetivos que el Gobierno autónomo socialista se había marcado con relación a la modernización de la región y la necesidad de conectar ésta con los ejes más dinámicos del país y de Europa. En total dicha Consejería absorbió 84.092 millones de pesetas, es decir, el $24,7 \%$. Sanidad y Servicios Sociales acaparó otra buena parte de los Presupuestos, 69.836 millones de pesetas que representan el 20,5\%. Más alejadas de este volumen aparecen las Consejerías de Medio Rural y Pesca (13,8\%) y Hacienda, Economía y Planificación (12,5\%). La Consejería con un presupuesto más pequeño es Interior y Administraciones Públicas $(3,2 \%)$ ya que su cometido es de gestión interna y administración.

El cambio político antes señalado se acompañó de una reestructuración de las 
Consejerías, cuyo número se reduce de ocho a seis (con la justificación por parte del nuevo Presidente de recortar gastos en la Administración) y cambian de denominación. Entre 1996 y 1998, la Consejería de Fomento, que incluye infraestructuras, urbanismo y vivienda, entre otros, sigue acaparando el grueso de los Presupuestos, con más del $44 \%$ del gasto, mientras se produce un trasvase paulatino de recursos desde la Consejería de Economía (que incluye las competencias relativas a la industria) hacia Agricultura (la primera pasa de 22,17\% del gasto a $12,74 \%$, mientras que la segunda evoluciona del $15,29 \%$ al $17,29 \%$ ) y en mucha mayor medida hacia Cultura, que duplica con creces su presupuesto: del $6,49 \%$ pasa al $14,79 \%$. Sin embargo, el giro más significativo en la política presupuestaria afecta a Sanidad y Servicios Sociales, con una participación en los Presupuestos que no supera el $3,4 \%$ en ninguno de los años considerados frente a un porcentaje superior al $20 \%$ en los años de la etapa precedente.

En cuanto a la contribución de los ingresos del Principado por fuentes de financiación, esta queda establecida de la siguiente forma:

- Participación del Estado: 19\%

- Porcentaje provincial: $15 \%$

- Tributos cedidos: $18 \%$

- Tributos propios: $6 \%$

- FCI: $4 \%$

- Fondos Estructurales: 9\%

- Endeudamiento: $11 \%$

- Otros ingresos: $17 \%$

De tanto o mayor interés para profundizar en el conocimiento de la relación poder-espacio es el análisis territorial de los Presupuestos, es decir, el reparto de la inversión de la Administración regional por comarcas. Como es sabido, la distribución territorial de la actividad económica en Asturias se caracteriza por su concentración en la zona central, en los municipios de Oviedo, Gijón, Avilés, Mieres y Langreo, que a su vez concentran la mayoría de la población, de las infraestructuras y de los servicios públicos. En el resto de la región, en las zonas oriental y occidental, la población y las activides productivas son mucho menores tanto en términos absolutos como relativos, contraponiendo su carácter rural al industrial y de servicios de la zona central (BENITO DEL POZO, 1995).

Según los argumentos de la propia Administración del Principado, la situación de desequilibrio territorial que acaba de apuntarse, unida a la precaria situación de la industria, hacen necesario que los Presupuestos regionales busquen tanto un efecto reequilibrador en favor de las comarcas periféricas, como promover las medidas y actuaciones adecuadas para propiciar la recuperación industrial de las comarcas centrales.

En términos absolutos, en los distintos años del período 1995-98 la inversión total se repartió con preferencia entre las comarcas de Oviedo, Gijón, Nalón y Avilés, es decir entre las comarcas siderúrgicas y mineras de la zona central (añadiendo el Caudal), a su vez la más poblada de la región; las comarcas de la zona occidental (Eo-Navia y Narcea) recibieron la segunda porción más abultada del total del Presupuesto, quedando el Oriente como la comarca con menos gasto asignado en correspondencia a su menor población y extensión geográfica. En 
términos relativos, la inversión alcanzada por la zona central oscila, según los años, entre el $58 \%$ y el $61 \%$, mientras que la zona occidental absorbe entre el $26 \%$ y el $28 \%$ y la oriental entre el $7 \%$ y el $12 \%$ (CUADRO 1 ).

Como tendencias, la zona central sigue y seguirá siendo la destinataria principal del grueso de los Presupuestos, pues en los últimos años considerados gana participación en los mismos, lo que contradice los objetivos políticos del partido en el poder de orientar la política regional en sentido de favorecer a las comarcas periféricas y buscar el necesario equilibrio territorial que atenúe contrastes entre el centro y las "alas" de la región. La lógica de concentrar recursos allí donde se concentran población y problemas industriales se impone ante el discurso político de equilibrar y apoyar el desarrollo paralelo de las comarcas periféricas, hecho en absoluto extraño en la dialéctica que relaciona poder y territorio, voluntad política proclamada y práctica del poder.

Cuadro 1. Inversión (miles de pesetas) y población por comarcas (19951998).

\begin{tabular}{lrrrr}
\hline Comarcas & Poblac. 1991 & Inversión 1996 & Inversión 1997 & Inversión 1998 \\
\hline Eo-Navia & 60.418 & $6.549 .612,8$ & $7.274 .177,4$ & $7.591 .274,1$ \\
Narcea & 41.187 & $5.805 .687,5$ & $5.678 .755,8$ & $6.717 .500,7$ \\
Avilés & 163.539 & $7.787 .150,8$ & $8.954 .888,9$ & $10.991 .421,4$ \\
& & & & \\
Oviedo & 307.308 & $16.649 .826,2$ & $16.261 .551,5$ & $19.632 .527,8$ \\
& & & & \\
Gijón & 285.174 & $12.345 .715,8$ & $11.828 .367,1$ & $11.596 .422,8$ \\
& & & & \\
Caudal & 85.155 & $5.437 .547,5$ & $5.111 .808,6$ & $5.035 .301,6$ \\
Nalón & 93.642 & $8.579 .516,8$ & $6.517 .477,3$ & $6.964 .424,5$ \\
Oriente & 57.514 & $7.222 .633,1$ & $7.335,163,1$ & $7.434 .520,8$ \\
Asturias & 1.093 .937 & $70.377 .691,0$ & $68.962 .190,0$ & $75.963 .394,0$ \\
& & & & \\
\hline
\end{tabular}

FUENTE: Presupuestos Generales del Principado de Asturias, varios años.

Con respecto al destino de las inversiones, observamos que desde 1995 se concede gran importancia a las infraestructuras que articulan el territorio, a la construcción de viviendas y a la dotación de equipamientos que mejoren la calidad de vida de los habitantes de la región; al mismo tiempo, parte del esfuerzo inversor se orienta a la promoción económica, a fin de estimular la reindustrialización de la zona central (Presupuestos Generales del Principado de Asturias, varios años).

\section{PLANIFICACIÓN REGIONAL Y ESPACIAL.}

La planificación del desarrollo es una tarea básica de las Administraciones públicas y un asunto de interés para el geógrafo que busca comprender la dimensión económica del territorio. El Principado tiene como marco general para establecer la estrategia de planificación de Asturias y dar coherencia y coordinar las 
numerosas actuaciones requeridas y ofrecer con la suficiente antelación una proyección a medio plazo de las prioridades del Gobierno regional, el denominado Plan de Desarrollo Regional $(P D R)$. El primer Plan cubría el período 19851989, el segundo el período 1989-1993 y el tercero, con vigencia actual, se extiende desde 1994 hasta 1999.

El objetivo general de dicho instrumento es colaborar en la sustitución del modelo económico que aún persiste en Asturias (fuerte especialización en producciones básicas recesivas y concentración espacial de las mismas) por otro alternativo que posibilite el logro de un sistema productivo regional diversificado, más competitivo y equilibrado, tanto sectorial como territorialmente, y ello con la meta de lograr un nivel de desarrollo económico que permita incrementar el nivel de bienestar de la población que se asienta en la región.

En consecuencia, la política de promoción y desarrollo que define el PDR en vigor tiene dos objetivos estratégicos: modernización y mejora de la competitividad del tejido existente, y diversificación y ampliación de ese tejido. A tal fin los recursos financieros previstos para el periodo $1994-99$ ascienden a $311.700 \mathrm{mi}-$ llones de pesetas.

Otro instrumento importante es el Programa de Reindustrialización y Dinamización Económica de Asturias, 1994-1999, ejemplo a su vez de la colaboración entre el Principado y la Administración del Estado. Consiste en un conjunto de acciones orientadas a crear las condiciones que permitan regenerar el tejido industrial y empresarial de la región, poniendo en juego todos los elementos necesarios para que la iniciativa privada pueda, en colaboración con la inversión pública, afrontar con garantías el futuro socioeconómico de Asturias. Los recursos públicos previstos ascienden a 97.191 millones de pesetas para 1994-1995 y a 296.839 para el período 1996-1999. Las grandes líneas de acción son infraestructuras, medio ambiente, promoción económica, formación y agricultura y pesca, aspectos todos relacionados con el relanzamiento de la economía regional y con lo que se entiende por calidad de vida.

En el ámbito de la planificación espacial cabe destacar dos líneas de intervención fundamentales: la ordenación del territorio y la planificación urbanística. Desde finales de los años ochenta, los poderes públicos y otros agentes sociales tomaron conciencia de la necesidad de intervenir en ámbitos espaciales que superasen el marco exclusivo de la ciudad y del municipio. Surge así una propuesta de planeamiento supramunicipal que toma como referencia legal no la legislación urbanística que rige para los primeros, sino las nuevas leyes de Ordenación del Territorio con que se dotan las Comunidades Autónomas (UREÑA, 1997).

Este giro conceptual sobre el planeamiento es, en gran medida, reflejo de las nuevas realidades que van tomando fuerza en nuestro país desde los años citados: la formación de áreas metropolitanas que precisan instrumentos nuevos para la ordenación del espacio, y la creación de ámbitos regionales con autonomía política y administrativa que requieren una ordenación específica. A escala autonómica merecen especial referencia las Directrices Regionales de Ordenación del Territorio, un instrumento de planificación flexible concebido para superar los esquemas jerárquicos de la Ley del Suelo y que hace necesaria una Ley pre- 
via de Coordinación y Ordenación que defina los principios e instrumentos básicos para dicha ordenación (LÓPEZ TRIGAL Y BENITO DEL POZO, 1999), que en el caso de Asturias es la Ley 4/1987, de 30 de marzo, de Coordinación y Ordenación Territorial.

Así concebidas, las Directrices se constituyen en el principal elemento de planificación y coordinación territorial y la base para el desarrollo de las actuaciones con impacto que hayan de producirse en cada Comunidad Autónoma. Dicha figura en Asturias se enmarca en el bloque programático referido a la mejora del medio físico, la ordenación del territorio y las comunicaciones, tomando como parámetros básicos los siguientes presupuestos:

a) Se debe producir un cambio cualitativo en la programación regional del territorio, pues ni los planeamientos urbanísticos municipales ni la superposición de las diferentes políticas sectoriales aplicadas en la región son suficientes para definir un sistema adecuado de relaciones espaciales y de uso del territorio.

b) La programación regional del territorio debe ser un elemento de contraste y referencia de las demás políticas.

No existe una metodología única para abordar procesos de ordenación territorial, por lo que cada Comunidad Autónoma que se plantea redactar un documento de esta naturaleza aplica un método diferente acorde con la problemática territorial y los rasgos socioeconómicos que le son propios, si bien en todos los casos se parte de un diagnóstico preliminar a partir del cual se definen los criterios que habrán de orientar la ordenación del territorio y el proceso de redacción de las Directrices.

El objetivo fundamental de las Directrices asturianas es, ante la evidencia de los fuertes desequilibrios territoriales entre el centro y las periferias regionales, propiciar el reequilibrio territorial sobre la base de que la zona central ha de actuar como motor capaz de inducir el desarrollo de las zonas oriental y occidental mediante la descentralización de actividades industriales y terciarias hacia la periferia. Se trata de evitar la compactación del área central en favor de la periferia, conjugando los objetivos económicos y territoriales en el área central y en el conjunto de la región. Los instrumentos para su desarrollo son las Directrices Subregionales, de ámbito supracomarcal (Área Central, Zona Oriental, Zona Occidental, y Franja Costera), las Directrices Sectoriales y los Programas de Actuación Territorial.

La segunda línea de intervención de los poderes públicos en el territorio viene expresada por la planificación urbanística. Como señalan TRIGAL Y BENITO (1999), "el planeamiento es la clave de la ordenación urbanística en la medida en que es el instrumento técnico de diseño territorial y el instrumento político de programación de las actividades públicas y privadas en orden a la realización del modelo urbano". A escala municipal están los Planes Generales Municipales de Ordenación y las Normas Subsidiarias, los primeros más complejos y completos que las segundas al incluir los Sistemas Generales, es decir, los elementos al servicio de toda o de la mayoría de la población (calles, plazas, jardines...) y un Programa de Actuación que fija el desarrollo de las actuaciones previstas por el Plan. Los municipios pequeños, con problemas urbanísticos de poca envergadura suelen optar por las Normas Subsidiarias. 


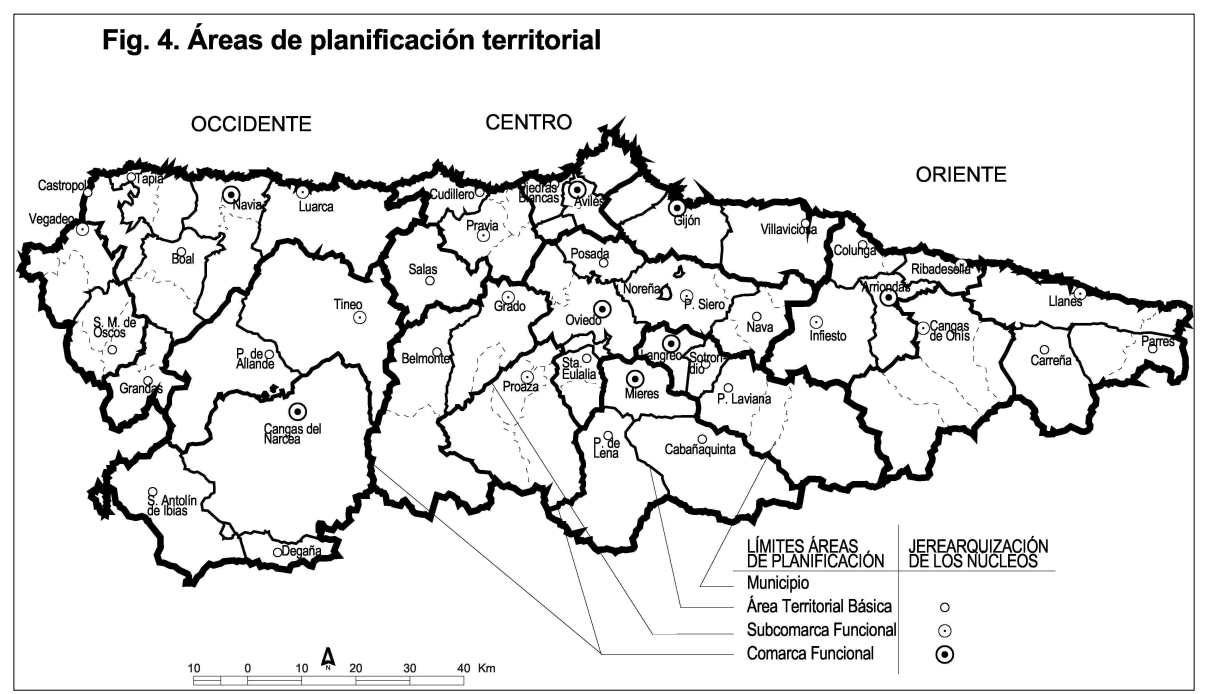

En la evolución de la planificación urbanística reciente en España es posible distinguir dos etapas bien diferenciadas: los años de planeamiento anteriores a la transición política y la etapa democrática. Los primeros tienen como eje la Ley del Suelo de 1956 y se caracterizan por el ejercicio de un planeamiento expansivo, fundado en la idea de una ciudad que tiene que crecer y ser ampliada, lo que obliga a la habilitación de suelo sobre el que producir las nuevas piezas de la trama urbana, en condiciones de fuerte especulación y ante la actitud pasiva de los Ayuntamientos (TERÁN, 1996; UREÑA, 1997).

La constitución de Ayuntamientos democráticos a partir de 1979 y la aprobación de una nueva Ley del Suelo unos años antes sientan las bases para un planeamiento más participativo y eficaz desde el punto de vista instrumental, al tiempo que surgen nuevos enfoques y actitudes entre los responsables públicos. Pero el cambio no es uniforme en todos los municipios. Según UREÑA (1997), en los municipios con gobiernos de izquierdas se presta atención prioritaria a las deficiencias en equipamientos, transportes, urbanización y servicios en atención a las demandas vecinales con intención de mejorar la calidad y estructuración de la ciudad existente; se impone, en definitiva, el entendimiento del Plan como "proyecto de ciudad". Sin embargo en municipios con gobiernos similares a los predemocráticos sigue primando el Plan enfocado hacia la expansión de la ciudad, más pendiente de los intereses particulares que de los generales.

En la actualidad, la planificación urbanística comporta la puesta a punto de nuevos instrumentos que incluyan aspectos de previsión ligados al futuro de las ciudades, tales como la referencia al marco regional o la organización estructural del complejo urbano-territorial en función de la demanda del transporte. Además, el planeamiento es cada vez más sensible al impacto de otros instru- 
mentos de desarrollo de la ciudad que toman en consideración aspectos urbanísticos, como es el caso de la planificación estratégica en su comprensión integral de la realidad urbana (TRIGAL Y BENITO, 1999).

Asturias puede considerarse una región avanzada en materia de planificación urbanística. Pocos son los municipios que carecen de un instrumento propio en esta materia (en las distintas fases de aprobación que son preceptivas para su tramitación), en su mayoría Normas Subsidiarias adaptadas al pequeño tamaño de los municipios y la escasa entidad de la mayoría de las poblaciones. Sólo siete municipios, todos situados en el área central y siendo parte integrante del área metropolitana en formación, poseen un Plan General: Oviedo, Avilés, Gijón, Mieres, Langreo, Siero y Castrillón. La situación descrita se traduce en un control relativamente efectivo por parte de los agentes públicos sobre el territorio en materia urbanística, lo que evita o atenúa los excesos especulativos (no siempre ni fácilmente controlables).

\section{PROVISIÓN DE SERVICIOS PÚBLICOS.}

Por lo que respecta a la provisión de servicios públicos como la educación y la enseñanza, la sanidad, los servicios sociales, el medio ambiente y los instrumentos regionales de promoción económica, el Principado de Asturias ofrece un nivel de cobertura aceptables, aunque hay diferencias entre unos servicios y otros y, sobre todo, todavía se está lejos de una equitativa distribución territorial del conjunto de todos ellos. La tendencia es a descentralizar, aunque este proceso es imperfecto, entre otras razones por la falta de competencias, la descoordinación entre los distintos niveles de la Administración y, en ocasiones, por la limitación de los recursos disponibles. Los esfuerzos (políticas, acciones y financiación) de los responsables públicos en esta materia están reforzados por la evidencia de que son elementos de calidad de vida y éste es un factor de dinamización económica e integración territorial que contribuye de forma sustancial al logro de otros objetivos como el crecimiento, la diversificación económica o la formación.

\section{1) Educación}

El Principado tiene competencias parciales en la educación reglada, sólo en enseñanza superior. La Universidad de Oviedo concentra prácticamente la totalidad de la enseñanza universitaria de la región, alcanzando un número de alumnos superior a 40.000. Esta cifra de alumnos no se reparte de forma igualitaria entre todas las titulaciones. Así por ejemplo, el mayor número de ellos se concentra en las carreras de ciencias sociales, aunque las que han alcanzado un crecimiento mayor del alumnado en los últimos años son las carreras técnicas de ciclo corto. Desde el punto de vista espacial destaca la existencia de tres campus: Oviedo, Gijón y Mieres. Sólo Oviedo posee facultades, además de escuelas técnicas superiores, escuelas universitarias y escuelas profesionales, siendo el desarrollo de este campus muy superior a los otros dos.

Como medio de responder a la demanda social, la Universidad de Oviedo está en período de transformación de sus titulaciones, ofertando nuevos títulos y adaptando numerosos planes de estudios para darles un mayor carácter práctico, 
lo que se intenta reforzar con la firma de contratos de colaboración entre los distintos centros universitarios y las empresas. También está sujeta a un proceso de dispersión geográfica (ampliación de los campus de Gijón y Mieres) con lo que se trata de dar respuesta a los problemas económicos de las ciudades más castigadas por la crisis industrial, que buscan en el desarrollo de la función universitaria una alternativa a las actividades productivas tradicionales.

En cuanto a la enseñanza secundaria y primaria, ambas están en transformación, como ocurre en el resto del Estado, debido a la aplicación de la LOGSE, que presta especial atención a la formación profesional y a la ordenación y mejora de calidad de la escuela rural. La reforma educativa trata de aproximarse al medio ofertando estudios ligados a las actividades económicas de la zona donde se encuentra el centro, como vía para motivar a los alumnos.

La aplicación de la LOGSE supone, también, un cambio importante en la Formación Profesional (FP), tanto en sus estudios como en los objetivos propuestos. Una de las características más novedosas de la nueva FP es la inclusión de un área de formación en centros de trabajo con carácter obligatorio, así como el aumento del número de especialidades, lo que se interpreta como un intento de adaptación a las nuevas exigencias del mercado laboral.

Sobre la distribución geográfica de los centros de enseñanza secundaria y primaria, es importante señalar que mientras la primaria tiene una distribución más regular y uniforme por todo el territorio regional, la secundaria tiende a localizarse en lugares centrales, hecho que impone un desplazamiento diario de ida y vuelta a los alumnos alejados del centro y la necesidad de poner en marcha un servicio regular de transporte colectivo que comunique los distintos puntos de residencia de los alumnos con el centro escolar.

En cuanto a la enseñanza no reglada, Formación Ocupacional (FO), la oferta pública está canalizada por dos organismos: el INEM y el Principado de Asturias, y se concreta en la creación de escuelas-taller y casas de oficios, principalmente. El conjunto de la oferta de Fo pública ha experimentado desde 1984 un incremento progresivo inercial, que alcanzó su momento álgido en 1990-91 y que se ha reducido ligeramente en los últimos años. Este crecimiento ha generado toda una "industria" pública y semipública de la formación compuesta por una multitud de participantes.

\section{2) Sanidad}

En servicios de salud, Asturias dispone de pocas competencias. El Principado disfruta de competencias sobre el Servicio de Salud Mental, el Hospital Monte Naranco y el Hospital General de Asturias. Este último está funcionalmente integrado en el complejo hospitalario de Oviedo, junto con el Hospital Covadonga (antigua Residencia Sanitaria) y en Instituto Nacional de Silicosis, denominados todos estos servicios Hospital Central de Asturias. Existe un convenio entre el Principado y el Ministerio de Sanidad para la gestión compartida del Hospital Central. Asturias también tiene competencias en materia de planificación y ordenación sanitaria, aspecto este último de sumo interés puesto que la elaboración del mapa sanitario determina la ordenación territorial y disposición de los servicios de salud a través de la delimitación de las Áreas de Salud y las Zonas de Salud. 
Una vez transferidas las competencias, aún sin fecha precisa, la gestión pasará a un organismo, ya constituido, denominado Servicio de Salud del Principado de Asturias (SESPA) y adscrito a la Consejería de Sanidad y Servicios Sociales, que mantiene una relación similar a la que se establece a nivel central entre el Ministerio de Salud y el INSALUD.

Entre los problemas detectados en los servicios de salud cabe destacar que en 1991 algo más del 40\% de la población asturiana no estaba cubierta por el nuevo modelo de atención primaria. Por otro lado, han disminuido los valores de cama por habitante, debido a causas como que los hospitales del INSALUD aumentan su oferta mientras que las camas del sector privado se reducen, así como las camas psiquiátricas debido a la estructuras del sistema de salud mental.

Respecto a la accesibilidad geográfica, ésta parece aceptable. El 98,7 por ciento de la población tiene un dispositivo de atención primaria a menos de 30 minutos de su domicilio. Esta cifra debe considerarse como muy aceptable en una región de orografía difícil y población dispersa. Este mismo indicador es del 97,3 por ciento para la atención urgente y del 85,1 por ciento para la atención especializada.

3) Servicios Sociales

El sistema de servicios sociales se materializa en una red de centros municipales que sirven prácticamente al 100 por ciento de la población asturiana. La puesta en marcha de esta red se realizó en el marco del Convenio Programa con el Ministerio de Asuntos Sociales para el desarrollo de prestaciones de Servicios Sociales en las Corporaciones Locales a partir de la cooperación de las Administraciones central, autonómica y local. Existen 37 centros de Servicios Sociales, cuyos programas han supuesto, en los años 1991-1994 un coste superior a 1.800 millones de pesetas, para promover y posibilitar el bienestar social.

Además de estos servicios de atención básicos, hay otros especializados dirigidos a sectores de la sociedad que por sus peculiaridades requieren una atención diferenciada:

- Plan de Atención al Anciano.

- Desarrollo de la Ley de Protección del Menor.

- Plan de Minusvalías.

- Plan Concertado para el Desarrollo de los Servicios Sociales.

- Ingreso Mínimo de Inserción.

- Plan General de Inserción del Principado.

- Plan Integral de Juventud.

Los retos de esta política social del Principado serán el fomento de acciones dirigidas a los colectivos más desfavorecidos, la consolidación del sistema de prestaciones sociales básicas, a través de los centros de Servicios Sociales, así como del Ingreso Mínimo de Inserción para personas carentes de recursos; la completa integración del anciano y de los menores en situación de desamparo, y en las áreas de juventud y mujer, la promoción de la plena integración y participación de ambos colectivos en la vida comunitaria, social y cultural, apoyando el asociacionismo y la protección pública de los mismos.

4) Medio Ambiente

Asturias es, desde el punto de vista medioambiental, una sociedad de contras- 
tes: por una parte ha conservado ciertas zonas de la región con calidad medioambiental y paisajística muy alta, y por otro lado impulsado actividades productivas en extremo perjudiciales para el medio natural.

En los últimos años la concienciación sobre el deterioro y a la vez los valores del medio ambiente ha sido notable. El medio ambiente ha pasado a considerarse un recurso que genera importantes beneficios y por tanto que hay que cuidar.

La política medioambiental asturiana se sitúa en tres frentes: agua, contaminación atmosférica y residuos sólidos. En el primer caso los proyectos más importantes han sido la creación del consorcio CADASA en el año 1976 con el fin de resolver el problema de la escasez de abastecimiento de agua en la zona central de la región, problema que revestía especial gravedad y urgencia por cuanto no sólo se trataba de la comarca con mayor densidad de la región, sino además la más industrializada; y la puesta en marcha del Plan Regional de Infraestructura Hidráulica, cuyo fin básico es solucionar los problemas en materia de saneamiento y contaminación de las aguas.

Para detectar la contaminación atmosférica existe una red de medición y control de la contaminación atmosférica en Asturias que ha permitido el estudio de los niveles de contaminación por partículas en suspensión, dióxido de azufre y en algunos lugares óxidos de nitrógeno. Las medias diarias de todos los sensores de la red que superan los valores guía recomendados (establecidos para proteger la salud de las personas más sensibles) pueden utilizarse como medida de calidad del aire que respiramos (valores de inmisión). En el Principado el mayor problema lo presentan las partículas en suspensión que en Langreo suelen sobrepasarse en más de un 20 por ciento de los días, colocándose a continuación los valores de Gijón, Oviedo y por último Avilés.

Sobre los residuos sólidos destaca, por último, la creación del Consorcio para la Gestión de Residuos Sólidos en Asturias (COGERSA). En el año 1982, ante el inmediato agotamiento de los vertederos que venían utilizando varios concejos de Asturias con mayor producción de residuos sólidos urbanos, entre ellos Gijón, Oviedo y Avilés, éstos decidieron crear, con el apoyo del Principado, un Consorcio que les permitiese solucionar de forma conjunta la eliminación de sus residuos y que se ha ido extendiendo por la zona central.

\section{5) Instrumentos de promoción económica}

Los graves problemas económicos de la región, donde en el transcurso de la última década, con especial intensidad, se ha desmantelado una parte sustancial de su tejido industrial, ha llevado a la Administración del Principado a desarrollar una creciente labor de promoción económica dirigida tanto a proyectos asturianos como a captar inversiones procedentes del exterior. A tal fin se ha creado una red de organismos que apoya y promueve la creación de nuevas empresas, sin que los resultados de sus actividades hayan logrado romper el círculo del declive prolongado que caracteriza a la región desde que se produjese la reconversión a la baja de sus principales sectores y empresas productivas.

Según el análisis de CASTELLS (1994), la base institucional es muy compleja, poco coordinada y con una reducida capacidad de incorporación del empresariado. Existen pluralidad de organismos que realizan actuaciones similares sin la coordinación adecuada. Las duplicaciones más importantes se constatan entre el 
Instituto de Fomento Regional (IFR) y el Servicio de Asesoramiento y Promoción Empresarial (SAYPE), ambas entidades con los presupuestos más elevados; o entre la Sociedad Regional de Promoción (SRP) y la Sociedad para el Desarrollo de las Comarcas Mineras (SODECO), dos sociedades de capital riesgo.

La política futura en este ámbito de intervención de los poderes públicos pasa, a nuestro juicio, por una ordenación de la base institucional de las actuaciones y porque cada organismo tenga definidas claramente sus estrategias, amén de una colaboración más estrecha entre todas las instancias implicadas en el desarrollo de la región.

\section{CONCLUSIONES.}

El poder político y el territorio son, como hemos tratado de demostrar, elementos que se interrelacionan hasta el punto que el proyecto de administración y gestión que es inherente al primero no puede realizarse sin el concurso del segundo, a su vez muy sensible en su articulación y dinámicas a las distintas políticas sectoriales y de intervención directa. Cómo se defina esa relación y se concrete en actuaciones y resultados dependerá de las distintas concepciones que desde el poder se tengan sobre el modelo económico, social y territorial a desarrollar. Hay en ello un fuerte componente ideológico y también condicionantes externos, como son la base económica y espacial de partida, que, sin embargo, son moldeables dentro de ciertos márgenes y adaptables al modelo que se pretende imponer desde los ámbitos de decisión política.

En Asturias, una región de tradición industrial en declive, muy dependiente en su proceso de recuperación económica y social de las ayudas del sector público, la intervención de las Administraciones central y regional resulta imprescindible y determinante para corregir las tendencias de retroceso, frenar la emigración y paliar los fuertes desequilibrios territoriales entre las tres grandes zonas de la región. Hasta qué punto los poderes públicos son capaces de activar instrumentos que permitan hacer realidad esos objetivos es algo que hemos querido poner de relieve en este trabajo aunque sea, siquiera, de forma aproximada. Los resultados evaluados no permiten ser, por el momento, demasiado optimistas: los desequilibrios territoriales persisten, la articulación del territorio es imperfecta y la integración entre comarcas es aún débil.

\section{FUENTES Y BIBLIOGRAFÍA.}

- Asturias 1991-1995, cuatro años para el futuro, Servicio de Publicaciones del Principado de Asturias, Oviedo, 1995.

- BENITO Del Pozo, P. (1993): Estrategias territoriales para el desarrollo económico de Asturias, Programa ERA, Documento de Trabajo nº 24, Principado de Asturias, Oviedo (mímeo).

- Benito Del Pozo, P. (1995) «La industria en Asturias: un declive prolongado», en J. BOSQUE MAUREL y R. MÉNDEZ: Cambio industrial y desarrollo regional en España, Oikos-Tau, Barcelona, pp. 113-146.

- Benito Del Pozo, P. (1997), «Los planes estratégicos en Asturias» en 
LÓPEZ TRIGAL, L. (dir.): El planeamiento urbano y estratégico. II Jornadas de Estudio y Debate Urbanos, Universidad de León, León, pp. 123-130.

- BOLETÍN OFICIAL DEL PRINCIPADO DE ASTURIAS, varios años.

- CASTELlS, M. (dir.) (1994): Estrategias para la reindustrialización de Asturias, Civitas, Madrid.

- CONSEJERÍA DE HACIENDA, ECONOMÍA Y PlanifiCACIÓN (1994): Plan de Desarrollo Regional de Asturias, 1994/1999, Oviedo (mímeo).

- CONSEJERÍA DE ORDENACIÓN DEL TERRITORIO, URBANISMO y MEDIO AMBIENTE (1987): Criterios para una demarcación territorial de Asturias, Servicio de Publicaciones del Principado de Asturias, Oviedo.

- CONSEJERÍA DE MEDIO AMBIENTE Y URBANISMO (1992): Directrices Regionales de Ordenación del Territorio, Servicio de Publicaciones del Principado de Asturias, Oviedo.

- FERNÁNDEZ GARCÍA, A. (1989): «Hacia la reorganización territorial de Asturias mediante las mancomunidades o uniones voluntarias de concejos», en Eria, $\mathrm{n}^{\mathrm{o}} 19-20$, pp. 175-194.

- LÓPEZ TRIGAL, L. (1984): «Límites, sedes y entidades territoriales en los Estatutos de Autonomía», en X Reunión de Estudios Regionales, León, AECR, vol. II, pp. 596-602.

- LÓPEZ TRIGAL, L. (1991): «Geografía y administración en España», en Boletín de la Asociación de Geógrafos Españoles, $\mathrm{n}^{\circ} 12$, pp. 19- 32 Madrid.

- LÓPEZ TRIGAL, L. (dir.)(1997): El planeamiento urbano y estratégico. II Jornadas de Estudio y Debate Urbanos, Universidad de León, León.

- LóPez Trigal, L.; Benito Del Pozo, P. (1999): Geografía Política, Cátedra, Madrid.

- MÉNDEZ, R.; MOLINERO, F.(1984): Geografia y Estado. Introducción a la Geografia Política, Cincel, Madrid.

- Presupuestos Generales del Principado de Asturias (varios años), Servicio de Publicaciones del Principado de Asturias, Oviedo.

- PRINCIPADO DE ASTURIAS (1992): Estatuto de Autonomía para Asturias, Servicio de Publicaciones, Oviedo.

- Quirós LinARES, F.(1993): División parroquial de Asturias, Servicio de Publicaciones del Principado de Asturias, Oviedo.

- SADEI (1991): Análisis de los servicios en Asturias, 1978-1990, Oviedo.

- SADEI: La renta de los municipios asturianos (varios años), Oviedo.

- SADEI: Datos y cifras de la economía asturiana (varios años), Oviedo.

- SADEI: Reseña estadística de los municipios asturianos (varios años), Oviedo.

- SÁnchez, J.-E. (1992): Geografía Política, Síntesis, Madrid.

- TAYLOR, P.J. (1994): Geografia Política, Trama Editorial, Madrid.

- TERÁN, F. de (1996): «Evolución del planeamiento urbanístico (18461996)», en Ciudad y Territorio. Estudios Territoriales, nº107-108, pp. 167-184.

- UREÑA, J.Ma . (1997): «Teoría y práctica urbanística» en L. LÓPEZ TRIGAL (dir.): El planeamiento urbano y estratégico, Servicio de Publicaciones de la Universidad de León, León, pp.13-24. 
RESUMEN: El presente artículo ofrece una aproximación a los asuntos que interesan a la nueva Geografía de la Administración, en el marco de las relaciones poder-espacio y bajo la premisa de que el territorio es protagonista o factor determinante de una serie de decisiones políticas que afectan de forma directa e inequívoca a la organización del espacio regional y a su dinámica social y económica. La singularidad del caso expuesto, Asturias, no impide extrapolar efectos de la intervención de los poderes públicos sobre el territorio que, sin duda, se reproducen en otras comunidades autónomas o regiones del país como consecuencia del común modelo administrativo.

PALABRAS CLAVE: Relaciones poder-espacio, Geografía Política, Geografía de la Administración, intervención en el espacio.

ABSTRACT: This paper tends towards the issues regarding the new Administration Geography within the framework of the relationships of power-space. This study takes into consideration the premise that territory is the main character or factor of a series of political decisions influencing, directly and unequivocally, the organization of the regional space and its social and economical action. The special nature of the case on display, Asturias, does not prevent from extrapolating the effects of the authorities intervention on territory which, undoubtedly, may recur on other self-governing regions of the country due to the common administrative model.

KEY WORDS: Relationships power-space, Political Geography, Administration Geography, intervention on territory.

RÉSUMÉ: Cet article offre une approximation des sujets qui intéressent la nouvelle Géographie de l'Administration, dans le cadre des rapports pouvoir-espace et sous la prémisse que le territoire est le protagoniste ou le facteur déterminant d'une série de décisions qui touchent de façon directe et évidente l'organisation de l'espace régional et sa dynamique sociale et économique. La singularité du cas exposé, les Asturies, n'empêche pas d'extrapoler les effets de l'intervention des pouvoirs publics sur le territoire, effets qui, sans aucun doute, se reproduisent dans d'autres communautés ou d'autres régions du pays comme conséquence du modèle administratif commun.

MOTS-CLÉS: Relations pouvoir-espace, Géographie Politique, Géographie de l'Administration, intervention dans l'espace. 